\title{
0-Hecke algebra actions on coinvariants and flags
}

\author{
Jia Huang $\|^{\dagger}$ \\ School of Mathematics, University of Minnesota, Minneapolis, MN, USA
}

\begin{abstract}
By investigating the action of the 0-Hecke algebra on the coinvariant algebra and the complete flag variety, we interpret generating functions counting the permutations with fixed inverse descent set by their inversion number and major index.

Résumé. En étudiant l'action de l'algèbre de 0-Hecke sur l'algèbre coinvariante et la variété de drapeaux complète, nous interprétons les fonctions génératrices qui comptent les permutations avec un ensemble inverse de descentes fixé, selon leur nombre d'inversions et leur "major index".
\end{abstract}

Keywords: 0-Hecke algebra, Ribbon number, Descent monomial, Demazure operator.

\section{Introduction}

A composition $I$ of an integer $n$ gives rise to a descent class of permutations in the symmetric group $\mathfrak{S}_{n}$; the cardinality of this descent class is known as the ribbon number $r_{I}$ and its inv-generating function is the $q$-ribbon number $r_{I}(q)$. Reiner and Stanton [15] defined a $(q, t)$-ribbon number $r_{I}(q, t)$, and gave an interpretation by representations of $\mathfrak{S}_{n}$ and $G L\left(n, \mathbb{F}_{q}\right)$.

Our main object here is to obtain similar interpretations of various ribbon numbers by representations of the 0-Hecke algebra $H_{n}(0)$ of type $A$. Norton [14] decomposed $H_{n}(0)$ into a direct sum of $2^{n-1}$ distinct indecomposable $H_{n}(0)$-submodules $M_{I}$ indexed by compositions $I$ of $n$. Consequently every indecomposable projective $H_{n}(0)$-module is isomorphic to $M_{I}$ for some $I$, and every simple $H_{n}(0)$ module is isomorphic to $C_{I}=\operatorname{top}\left(M_{I}\right)=M_{I} / \operatorname{rad} M_{I}$ for some $I$.

\subsection{Descent monomials and Demazure atoms}

Our first result is related to the descent monomials within $\mathbb{Z}[\mathbf{x}]=\mathbb{Z}\left[x_{1}, \ldots, x_{n}\right]$

$$
x_{w}=\prod_{i \in D(w)} x_{w(1)} \cdots x_{w(i)}, \quad w \in \mathfrak{S}_{n}
$$

\footnotetext{
${ }^{\dagger}$ Supported by NSF grant DMS-1001933.

1365-8050 @ 2011 Discrete Mathematics and Theoretical Computer Science (DMTCS), Nancy, France
} 
introduced by Garsia $[\overline{8}]$ as a $\mathbb{Z}$-basis for the coinvariant algebra $\mathbb{Z}[\mathbf{x}] /\left(\mathbb{Z}[\mathbf{x}]_{+}^{\mathfrak{S}_{n}}\right)$. The 0 -Hecke algebra $H_{n}(0)$ acts on the coinvariant algebra $\mathbb{Z}[\mathbf{x}] /\left(\mathbb{Z}[\mathbf{x}]_{+}^{\mathfrak{S}_{n}}\right)$ via the operators $\bar{\pi}_{i}=\pi_{i}-1$, where $\pi_{i}$ is the Demazure operator defined by

$$
\pi_{i} f=\frac{x_{i} f-s_{i}\left(x_{i} f\right)}{x_{i}-x_{i+1}} .
$$

Our first result shows that under this action:

- $\mathbb{Z}[\mathbf{x}] /\left(\mathbb{Z}[\mathbf{x}]_{+}^{\mathfrak{S}_{n}}\right)$ is isomorphic to $H_{n}(0)$ as a (left) $H_{n}(0)$-module;

- $\mathbb{Z}[\mathbf{x}] /\left(\mathbb{Z}[\mathbf{x}]_{+}^{\mathfrak{S}_{n}}\right)=\bigoplus_{I} N_{I}$, summed over all compositions of $n$, and $N_{I} \cong M_{I}$;

- $\mathbb{Z}[\mathbf{x}] /\left(\mathbb{Z}[\mathbf{x}]_{+}^{\mathfrak{S}_{n}}\right)$ has a $\mathbb{Z}$-basis of certain Demazure atoms whose leading terms under some order are the descent monomials.

Since $\mathbb{Z}[\mathbf{x}] /\left(\mathbb{Z}[\mathbf{x}]_{+}^{\mathfrak{S}_{n}}\right)$ has an extra grading by polynomial degree, it becomes a graded version of the left regular representation of $H_{n}(0)$.

\subsection{A bigraded characteristic}

Duchamp, Krob, Leclerc and Thibon [4] defined the characteristic of a finite dimensional $H_{n}(0)$-module $M$ with simple composition factors $C_{I_{1}}, \ldots, C_{I_{k}}$ to be

$$
\mathcal{F}(M)=\sum_{i=1}^{k} F_{I_{i}}
$$

where the $F_{I}$ 's are quasi-ribbon functions which form a basis for the algebra of quasi-symmetric functions. Krob and Thibon [10] showed that $\mathcal{F}\left(M_{I}\right)$ is the ribbon schur function $s_{I}$, and thus $\mathcal{F}(M)$ is symmetric whenever $M$ is projective.

If $M=H_{n}(0) v$ is cyclic then the length filtration

$$
H_{n}(0)^{(\ell)}=\bigoplus_{\ell(w) \geq \ell} \mathbb{Z} T_{w}
$$

induces a filtration of $H_{n}(0)$-modules $M^{(\ell)}=H_{n}(0)^{(\ell)} v, k \geq 0$. This refines $\mathcal{F}(M)$ to a graded characteristic

$$
\mathcal{F}_{q}(M)=\sum_{\ell \geq 0} q^{\ell} \mathcal{F}\left(M^{(\ell)} / M^{(\ell+1)}\right) .
$$

One has

$$
\mathcal{F}_{q}\left(H_{n}(0)\right)=\sum_{w \in \mathfrak{S}_{n}} q^{\operatorname{inv}(w)} F_{D\left(w^{-1}\right)}=\sum_{I} r_{I}(q) F_{I}
$$

and taking a limit as $q \rightarrow 1$ gives

$$
\mathcal{F}\left(H_{n}(0)\right)=\sum_{I} r_{I} F_{I}
$$


If $M$ has another filtration by $H_{n}(0)$-modules $M_{d}$ for $d \geq 0$, then one can look at the bifiltration by $H_{n}(0)$-modules $M^{(\ell, d)}=M^{(\ell)} \cap M_{d}$ for $\ell, d \geq 0$, and define the bigraded characteristic to be

$$
\mathcal{F}_{q, t}(M)=\sum_{\ell, d \geq 0} q^{\ell} t^{d} \mathcal{F}\left(M^{(\ell, d)} /\left(M^{(\ell+1, d)}+M^{(\ell, d+1)}\right)\right) .
$$

This happens to be the case for $M=\mathbb{Z}[\mathbf{x}] /\left(\mathbb{Z}[\mathbf{x}]_{+}^{\mathfrak{S}_{n}}\right)$ with its length filtration (since $M \cong H_{n}(0)$ ) and its polynomial degree filtration by $M_{d}=\langle\bar{f}: \operatorname{deg} f \geq d\rangle_{\mathbb{Z}}$ for $d \geq 0$. Our next result is

$$
\text { - } \mathcal{F}_{q, t}\left(\mathbb{Z}[\mathbf{x}] /\left(\mathbb{Z}[\mathbf{x}]_{+}^{\mathfrak{S}_{n}}\right)\right)=\sum_{w \in \mathfrak{S}_{n}} t^{\operatorname{maj}(w)} q^{\operatorname{inv}(w)} F_{D\left(w^{-1}\right)}
$$

which completes the following picture.

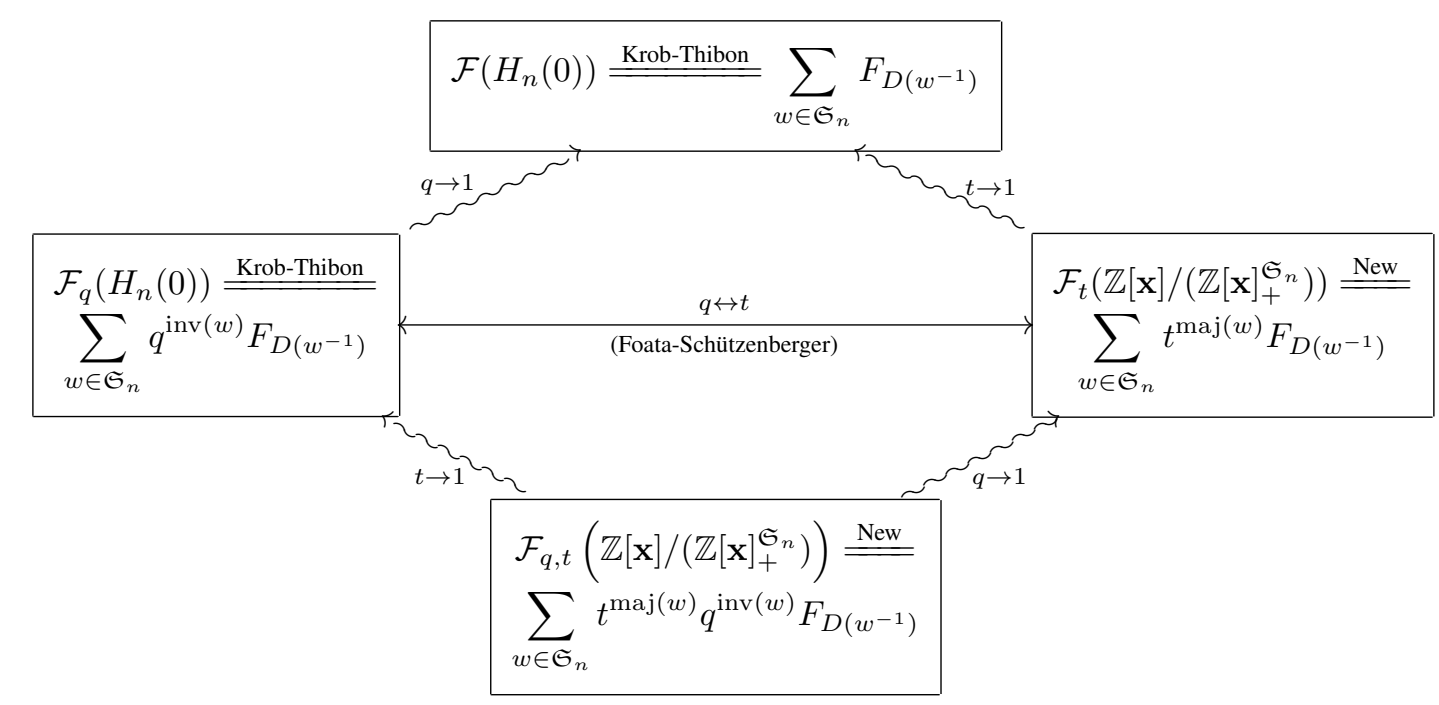

Here the left inverse descent set $D\left(w^{-1}\right)$ is identified with its descent composition, and the equality $\mathcal{F}_{q}\left(H_{n}(0)\right) \stackrel{q \leftrightarrow t}{=} \mathcal{F}_{t}\left(\mathbb{Z}[\mathbf{x}] /\left(\mathbb{Z}[\mathbf{x}]_{+}^{\mathfrak{S}_{n}}\right)\right.$ comes from the equidistribution of inv and maj over inverse descent classes, proved by Foata and Schützenberger [7]. We shall see in Section 3 that $r_{I}$ and $r_{I}(q)$ appear as coefficients of $F_{I}$ in $\mathcal{F}\left(H_{n}(0)\right)$ and $\mathcal{F}_{q}\left(H_{n}(0)\right)$.

\subsection{Complete flag variety}

Consider the general linear group $G=G L\left(n, \mathbb{F}_{q}\right)$ over a finite field $\mathbb{F}_{q}$ and its Borel subgroup $B$. The 0 -Hecke algebra $H_{n}(0)$ acts on the complete flag variety $1_{B}^{G}=\mathbb{F}_{q}[G / B]$ by $T_{w} B=B w B$. This action induces an $H_{n}(0)$-module structure on

$$
\operatorname{Hom}_{\mathbb{F}_{q}[G]}\left(1_{B}^{G}, \mathbb{F}_{q}[\mathbf{x}]\right) \cong \mathbb{F}_{q}[\mathbf{x}]^{B}
$$

which is $\mathbb{F}_{q}[\mathbf{x}]^{G}$-linear, hence inducing an $H_{n}(0)$-module structure on $\mathbb{F}_{q}[\mathbf{x}]^{B} /\left(\mathbb{F}_{q}[\mathbf{x}]_{+}^{G}\right)$. Our next result is: 
- $\mathcal{F}\left(1_{B}^{G}\right)=\sum_{I} r_{I}(q) F_{I}$;

- $\mathcal{F}_{t}\left(\mathbb{F}_{q}[\mathbf{x}]^{B} /\left(\mathbb{F}_{q}[\mathbf{x}]_{+}^{G}\right)\right)=\sum_{I} r_{I}(q, t) F_{I}$.

Therefore we have another picture as follows, which interprets all the ribbon numbers mentioned at the beginning.

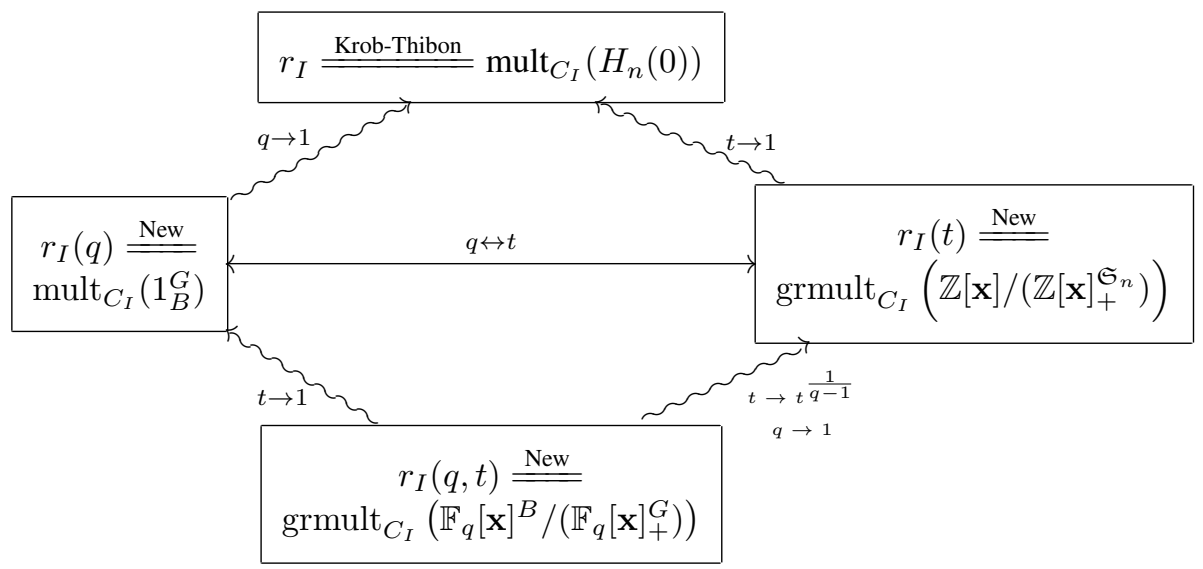

\subsection{Generalizations to other types}

The $H_{n}(0)$-actions on the coinvariants and complete flags can be generalized to the following setting. Let $W$ be a Weyl group with weight lattice $\Lambda$. The 0 -Hecke algebra $\mathscr{H}$ of type $W$ acts on the group ring $\mathbb{Z}[\Lambda]$ via the operators $\bar{\pi}_{i}=\pi_{i}-1$ where $\pi_{i}$ was originally considered by Demazure [2] in this setting. Garsia and Stanton [9] constructed the descent monomials in $\mathbb{Z}[\Lambda]$, which form a free basis over $\mathbb{Z}[\Lambda]^{W}$. We prove that, similarly to $\mathbb{Z}[\mathbf{x}] /\left(\mathbb{Z}[\mathbf{x}]_{+}^{\mathfrak{S}_{n}}\right)$, taking a quotient of $\mathbb{Z}[\Lambda]$ by the ideal generated by "positive" $W$-invariants leads to an $\mathscr{H}$-module isomorphic to $\mathscr{H}$ (without an extra grading). We also have an $\mathscr{H}$ action on $1_{B}^{G}$ by $T_{w} B=B w B$, where $G$ is a finite group with split $B N$-pair of characteristic $p>0$, whose Weyl group is $W$. We determine the characteristic $\mathcal{F}\left(1_{B}^{G}\right)$ in the same manner as for type $A$.

In Section 2 we review the definitions for the various ribbon numbers and their interpretation by representations of $\mathfrak{S}_{n}$ and $G L\left(n, \mathbb{F}_{q}\right)$. In Section 3 we recall the representation theory of the 0-Hecke algebra. The result for the coinvariant algebra $\mathbb{Z}[\mathbf{x}] /\left(\mathbb{Z}[\mathbf{x}]_{+}^{\mathfrak{S}_{n}}\right)$ is given in Section 4 , and a similar result for $\mathbb{Z}[\Lambda]$ of the weight lattice $\Lambda$ of a Weyl group is stated in Section 5 The $\mathscr{H}$-action on the complete flag variety $1_{B}^{G}$ and the coinvariant algebra of $(G, B)$ is investigated in Section 6 Lastly some questions are asked in Section 7

\section{Ribbon numbers}

We recall from Reiner and Stanton [15, $\S 9, \S 10]$ the definitions and properties of the various ribbon numbers. Let $I=\left(i_{1}, \ldots, i_{k}\right)$ be a composition of $n$, let $\sigma_{j}=i_{1}+\cdots+i_{j}$ for $j=1, \ldots, k$, and let the descent set of $I$ be $D(I)=\left\{\sigma_{1}, \ldots, \sigma_{k-1}\right\}$.

It is well known that compositions of $n$ bijectively correspond to the subsets of $[n-1]$ via their descent set; they also bijectively correspond to the ribbon diagrams, i.e. connected skew Young diagrams without $2 \times 2$ boxes, whose row sizes from bottom to top are $i_{1}, \ldots, i_{n}$. 
The descent class of $I$ is the set of all permutations $w$ in $\mathfrak{S}_{n}$ with $D(w)=D(I)$, and the inverse descent class is the set of all $w$ in $\mathfrak{S}_{n}$ with $D\left(w^{-1}\right)=D(I)$. The ribbon number $r_{I}$ is the cardinality of the descent class of $I$, and the $q$-ribbon number and $t$-ribbon number are

$$
\begin{aligned}
& r_{I}(q)=\sum_{w \in \mathfrak{S}_{n}: D(w)=D(I)} q^{\operatorname{inv}(w)}=[n] !_{q} \operatorname{det}\left(\frac{1}{\left[\sigma_{j}-\sigma_{i-1}\right] !_{q}}\right)_{i, j=1}^{k}, \\
& r_{I}(t)=\sum_{w \in \mathfrak{S}_{n}: D(w)=D(I)} t^{\operatorname{maj}\left(w^{-1}\right)}=[n] !_{t} \operatorname{det}\left(\frac{1}{\left[\sigma_{j}-\sigma_{i-1}\right] !_{t}}\right)_{i, j=1}^{k} .
\end{aligned}
$$

where $[n] !_{q}=[n]_{q}[n-1]_{q} \cdots[1]_{q}$ and $[n]_{q}=1+q+\cdots+q^{n-1}$. The notations make sense because $r_{I}(t)=\left.r_{I}(q)\right|_{q=t}$, which is a consequence of the equidistribution of inv and maj on every inverse descent class, proved by Foata and Schützenberger [7]. A further generalization is the $(q, t)$-ribbon number

$$
r_{I}(q, t)=n !_{q, t} \operatorname{det}\left(\varphi^{\sigma_{i-1}} \frac{1}{\left(\sigma_{j}-\sigma_{i-1}\right) !_{q, t}}\right)_{i, j=1}^{k}
$$

where $n !_{q, t}=\left(1-t^{q^{n}-1}\right)\left(1-t^{q^{n}-q}\right) \cdots\left(1-t^{q^{n}-q^{n-1}}\right)$, and $\varphi: t \mapsto t^{q}$ is the Frobenius operator.

All these ribbon numbers can be calculated by similar determinantal formulae, and are interpreted by certain homology representations $\chi^{I}$ of $\mathfrak{S}_{n}$, and $\chi_{q}^{I}$ of $G=G L\left(n, \mathbb{F}_{q}\right)$, together with their intertwiners

$$
\begin{gathered}
M^{I}=\operatorname{Hom}_{\mathbb{Z S}_{n}}\left(\chi^{I}, \mathbb{Z}[\mathbf{x}]\right), \text { a } \mathbb{Z}[\mathbf{x}]^{\mathfrak{S}_{n}} \text {-module, } \\
M_{q}^{I}=\operatorname{Hom}_{\mathbb{F}_{q} G}\left(\chi_{q}^{I}, \mathbb{F}_{q}[\mathbf{x}]\right), \text { an } \mathbb{F}_{q}[\mathbf{x}]^{G} \text {-module. }
\end{gathered}
$$

Here $\chi^{I}\left(\chi_{q}^{I}\right.$ resp.) is the top homology of the rank-selected Coxeter complex $\Delta\left(\mathfrak{S}_{n}\right)_{I}$ (Tits building $\Delta(G)_{I}$ resp.). Precisely, one has the following picture.

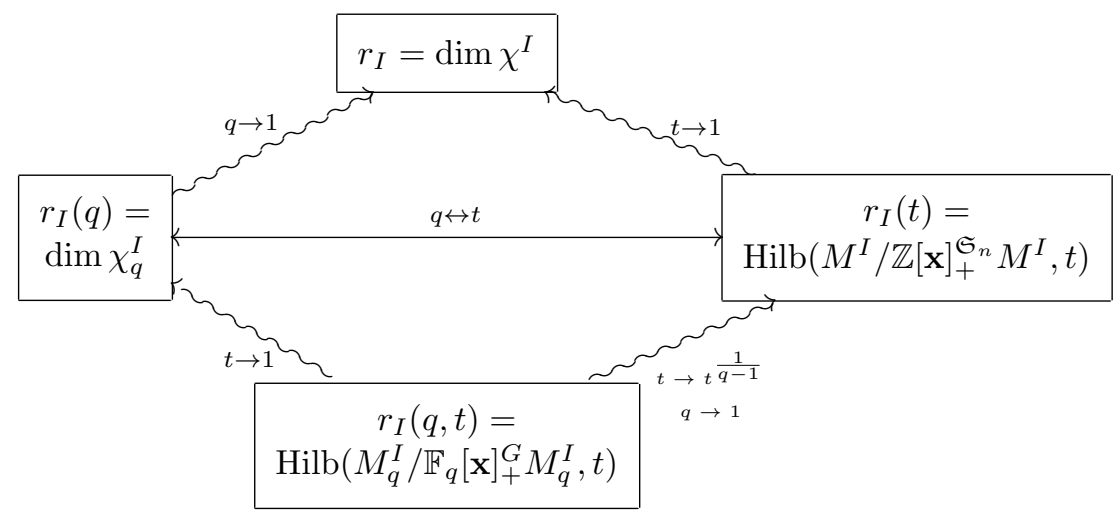

\section{Representation theory of the 0 -Hecke algebra}

Recall from Norton [14] the representation theory of the 0-Hecke algebra. Let

$$
W=\left\langle s_{1}, \ldots, s_{\ell}: s_{i}^{2}=1,\left(s_{i} s_{j} s_{i} \cdots\right)_{m_{i j}}=\left(s_{j} s_{i} s_{j} \cdots\right)_{m_{i j}}, 1 \leq i \neq j \leq \ell\right\rangle
$$


be a finite Coxeter group, where $(a b a \cdots)_{m}$ denotes an alternating product of $m$ terms. The 0-Hecke algebra $\mathscr{H}$ of type $W$ is an associative $\mathbb{Z}$-algebra generated by $T_{1}, \ldots, T_{\ell}$ with relations

$$
\begin{cases}T_{i}^{2}=-T_{i}, & 1 \leq i \leq \ell \\ \left(T_{i} T_{j} T_{i} \cdots\right)_{m_{i j}}=\left(T_{j} T_{i} T_{j} \cdots\right)_{m_{i j}}, & 1 \leq i \neq j \leq \ell .\end{cases}
$$

Norton [14] decomposed $\mathscr{H}$ into a direct sum of $2^{\ell}$ distinct indecomposable submodules $M_{I}$ indexed by compositions $I$ of $\ell+1$, with $C_{I}=\operatorname{top}\left(M_{I}\right)=M_{I} / \operatorname{rad} M_{I}$ being the (one-dimensional) simple module given by

$$
\rho_{I}\left(T_{i}\right)= \begin{cases}-1, & \text { if } i \in D(I) \\ 0, & \text { if } i \notin D(I)\end{cases}
$$

This gives a complete list of indecomposable projective $\mathscr{H}$-modules and simple $\mathscr{H}$-modules.

To explicitly construct $M_{I}$ inside $\mathscr{H}$, let $T_{i}^{\prime}=T_{i}+1,1 \leq i \leq \ell$. One can check that $\left(T_{i}^{\prime}\right)^{2}=T_{i}^{\prime}$, i.e. $T_{i} T_{i}^{\prime}=0$, and $\left(T_{i}^{\prime} T_{j}^{\prime} T_{i}^{\prime} \cdots\right)_{m_{i j}}=\left(T_{j}^{\prime} T_{i}^{\prime} T_{j}^{\prime} \cdots\right)_{m_{i j}}, 1 \leq i \neq j \leq \ell$; see [5, Lemma 3.1] or [14, Lemma 4.3]. Thus $T_{w}=T_{i_{1}} \cdots T_{i_{k}}$ and $T_{w}^{\prime}=T_{i_{1}}^{\prime} \cdots T_{i_{k}}^{\prime}$ are both well-defined if $w=s_{i_{1}} \cdots s_{i_{k}}$ is reduced.

Given a composition $I=\left(i_{1}, \ldots, i_{k}\right)$, let $\bar{I}=\left(i_{k}, \cdots, i_{1}\right)$ and let $I^{\sim}$ be the conjugate composition of $I$ obtained by reflecting the ribbon diagram of $I$ across the diagonal. For example, if $I=(2,1,3)$ then $\bar{I}=(3,1,2)$ and $I^{\sim}=(1,1,3,1)$. The descent class of $I$ is an interval $[\alpha(I), \omega(I)]$ in the left weak order of $W$, where $\alpha(I)$ is the top element in the parabolic subgroup $W_{D(I)}$. One can write the module $M_{I}$ in Norton's decomposition of $\mathscr{H}$ as $M_{I}=\mathscr{H} \cdot T_{\alpha(I)} T_{\alpha(\bar{I} \sim)}^{\prime}$, which has a $\mathbb{Z}$-basis given by

$$
\left\{T_{w} T_{\alpha(\bar{I} \sim)}^{\prime}: w \in[\alpha(I), \omega(I)]\right\} .
$$

\section{Coinvariant algebra of $\mathfrak{S}_{n}$}

The symmetric group $\mathfrak{S}_{n}$ acts naturally on the polynomial ring $\mathbb{Z}[\mathbf{x}]=\mathbb{Z}\left[x_{1}, \ldots, x_{n}\right]$ by permuting variables. The Demazure operators $\pi_{i}$ are defined by

$$
\pi_{i} f=\frac{x_{i} f-s_{i}\left(x_{i} f\right)}{x_{i}-x_{i+1}}
$$

where $s_{i}$ is the adjacent transposition $(i, i+1)$. One checks that the operators $\bar{\pi}_{i}=\pi_{i}-1$ satisfy the same relations as $T_{i}$, preserve the polynomial grading of $\mathbb{Z}[\mathbf{x}]$, and are $\mathbb{Z}[\mathbf{x}]^{\mathfrak{S}_{n}}$-linear. Thus one has an $H_{n}(0)$ action on the coinvariant algebra $\mathbb{Z}[\mathbf{x}] /\left(\mathbb{Z}[\mathbf{x}]_{+}^{\mathfrak{S}_{n}}\right)$ by sending $T_{i}$ to $\bar{\pi}_{i}$, or sending $T_{w}$ to $\bar{\pi}_{w}=\bar{\pi}_{i_{1}} \cdots \bar{\pi}_{i_{k}}$ if $w=s_{i_{1}} \cdots s_{i_{k}}$ is reduced.

One sees from the definition that $\bar{\pi}_{i}$ fixes all variables $x_{j}$ except $x_{i}$ and $x_{i+1}$, and

$$
\bar{\pi}_{i}\left(x_{i}^{a} x_{i+1}^{b}\right)= \begin{cases}x_{i}^{a-1} x_{i+1}^{b+1}+x_{i}^{a-2} x_{i+1}^{b+2} \cdots+x_{i}^{b} x_{i+1}^{a}, & \text { if } a>b, \\ 0, & \text { if } a=b, \\ -x_{i}^{a} x_{i+1}^{b}-x_{i}^{a+1} x_{i+1}^{b-1}-\cdots-x_{i}^{b-1} x_{i+1}^{a+1}, & \text { if } a<b .\end{cases}
$$

The module $\mathbb{Z}[\mathbf{x}]$ is free over $\mathbb{Z}[\mathbf{x}]^{\mathfrak{S}_{n}}$, with a basis consisting of the descent monomials

$$
x_{w}=\prod_{i \in D(w)} x_{w(1)} \cdots x_{w(i)}, w \in \mathfrak{S}_{n},
$$


constructed by Garsia [8]. We shall obtain a $\mathbb{Z}$-basis for the $H_{n}(0)$-module $\mathbb{Z}[\mathbf{x}] /\left(\mathbb{Z}[\mathbf{x}]_{+}^{\mathfrak{S}_{n}}\right)$ from certain Demazure atoms, i.e. $\bar{\pi}_{w} x_{I}$ for certain permutations $w$ and compositions $I$, where

$$
x_{I}=\prod_{i \in D(I)} x_{1} \cdots x_{i}
$$

See Mason [13] for more information on the Demazure atoms. The leading terms of these Demazure atoms are exactly the descent monomials, under any linear extension of the following partial order. Given two monomials $x^{d}$ and $x^{e}$, say $x^{d} \prec x^{e}$ if $\lambda(d)<_{L} \lambda(e)$ in the lexicographic order, where $\lambda(d)$ is the unique partition obtained from rearranging the exponent vector $d$, and similarly for $\lambda(e)$.

Lemma 4.1 Suppose that $I$ is a composition of $n$ and $w$ is a permutation in $\mathfrak{S}_{n}$ with $D(w) \subseteq D(I)$. Then

$$
\bar{\pi}_{w} x_{I}=w x_{I}+\sum_{d: x^{d} \prec x_{I}} c_{d} x^{d}
$$

where $c_{d} \in \mathbb{Z}$; moreover, $w x_{I}$ is a descent monomial if and only if $D(w)=D(I)$.

Theorem 4.2 The coinvariant algebra $\mathbb{Z}[\mathbf{x}] /\left(\mathbb{Z}[\mathbf{x}]_{+}^{\mathfrak{S}_{n}}\right)$ has a $\mathbb{Z}$-basis

$$
\left\{\bar{\pi}_{w} \bar{x}_{I}: w \in \mathfrak{S}_{n}, D(I)=D(w)\right\}
$$

and decomposes into a direct sum of $H_{n}(0)$-modules

$$
N_{I}=H_{n}(0) \cdot \bar{\pi}_{\alpha(I)} \bar{x}_{I}
$$

as $I$ runs through all compositions of $n$; moreover, $N_{I}$ is isomorphic to $M_{I} \subseteq H_{n}(0)$ and has a basis $\left\{\bar{\pi}_{w} \bar{x}_{I}: w \in[\alpha(I), \omega(I)]\right\}$. Thus $\mathbb{Z}[\mathbf{x}] /\left(\mathbb{Z}[\mathbf{x}]_{+}^{\mathfrak{S}_{n}}\right)$ is isomorphic to $H_{n}(0)$ as a left $H_{n}(0)$-module.

Since each submodule $N_{I}$ consists of homogeneous elements of degree maj $(w)$ for any $w \in[\alpha(I), \omega(I)]$, one has

$$
\mathcal{F}_{q, t}\left(\mathbb{Z}[\mathbf{x}] /\left(\mathbb{Z}[\mathbf{x}]_{+}^{\mathfrak{S}_{n}}\right)\right)=\sum_{w \in \mathfrak{S}_{n}} t^{\operatorname{maj}(w)} q^{\operatorname{inv}(w)} F_{D\left(w^{-1}\right)}
$$

which relates inv to the equidistributed maj (on every inverse descent class). In fact, one can get

$$
\mathcal{F}_{t}(\mathbb{Z}[\mathbf{x}])=\frac{1}{(1-t)\left(1-t^{2}\right) \cdots\left(1-t^{n}\right)} \sum_{w \in \mathfrak{S}_{n}} t^{\operatorname{maj}(w)} F_{D\left(w^{-1}\right)}
$$

directly from 11 using the filtration of $\mathbb{Z}[\mathbf{x}]$ induced from the following order (which appeared in Allen [1]): $x^{d}<_{t s} x^{e}$ if $\lambda(d)<_{L} \lambda(e)$, or if $\lambda(d)=\lambda(e)$ and $x^{d}<_{L} x^{e}$ in the lexicographic order. From (4) one can immediately deduce $\mathcal{F}_{t}\left(\mathbb{Z}[\mathbf{x}] /\left(\mathbb{Z}[\mathbf{x}]_{+}^{\mathfrak{S}_{n}}\right)\right)$, since $\bar{\pi}_{i}$ is $\mathbb{Z}[\mathbf{x}]^{\mathfrak{S}_{n}}$-linear.

\section{Coinvariant algebra of Weyl group}

Demazure's character formula [2] expresses the character of highest weight modules over a semisimple algebra using the Demazure operators $\pi_{i}$ on the group ring $\mathbb{Z}[\Lambda]$ of the weight lattice $\Lambda$. If $e^{\lambda}$ is the 
element in $\mathbb{Z}[\Lambda]$ corresponding to the weight $\lambda \in \Lambda$, and if $\lambda_{1}, \ldots, \lambda_{\ell}$ denote the fundamental weights with $z_{i}=e^{\lambda_{i}}$, then

$$
\mathbb{Z}[\Lambda]=\mathbb{Z}\left[z_{1}, \ldots, z_{\ell}, z_{1}^{-1}, \ldots, z_{\ell}^{-1}\right] .
$$

Suppose that $\alpha_{1}, \ldots, \alpha_{\ell}$ are the simple roots which correspond to simple reflections $s_{1}, \ldots, s_{\ell}$. Then the Demazure operators are defined by

$$
\pi_{i}=\frac{1-e^{-\alpha_{i}} s_{i}}{1-e^{-\alpha_{i}}}
$$

It follows easily that

$$
\pi_{i}\left(e^{\lambda}\right)= \begin{cases}e^{\lambda}+e^{\lambda-\alpha_{i}}+\cdots+e^{s_{i} \lambda}, & \text { if }\left\langle\alpha_{i}^{\vee}, \lambda\right\rangle \geq 0, \\ 0, & \text { if }\left\langle\alpha_{i}^{\vee}, \lambda\right\rangle=-1, \\ -e^{\lambda+\alpha_{i}}-\cdots-e^{s_{i} \lambda-\alpha_{i}}, & \text { if }\left\langle\alpha_{i}^{\vee}, \lambda\right\rangle<-1\end{cases}
$$

See, for example, Kumar [12]. Demazure operators satisfy the braid relation [2, 5.5] and

$$
s_{i} \pi_{i}=\pi_{i}, \pi_{i}^{2}=\pi_{i} .
$$

Hence the 0 -Hecke algebra $\mathscr{H}$ of $W$ acts on $\mathbb{Z}[\Lambda]$ by sending $T_{i}$ to $\bar{\pi}_{i}=\pi_{i}-1$.

Using the Stanley-Reisner ring of the Coxeter complex of $W$, Garsia and Stanton [9] showed that

$$
\mathbb{Z}[\Lambda]^{W}=\mathbb{Z}\left[a_{1}, \ldots, a_{\ell}\right]
$$

where

$$
a_{i}=\sum_{w \in W / W_{\{i\}^{c}}} e^{w \lambda_{i}}
$$

and $\mathbb{Z}[\Lambda]$ has a free basis over $\mathbb{Z}[\Lambda]^{W}$, which consists of the descent monomials

$$
z_{w}=\prod_{i \in D(w)} e^{w \lambda_{i}}, w \in W
$$

(see also Steinberg [17]). This basis induces a $\mathbb{Z}$-basis for $\mathbb{Z}[\Lambda] /\left(a_{1}, \ldots, a_{\ell}\right)$.

The $\mathscr{H}$-action on $\mathbb{Z}[\Lambda]$ is $\mathbb{Z}[\Lambda]^{W}$-linear, hence inducing an $\mathscr{H}$-action on $\mathbb{Z}[\Lambda] /\left(a_{1}, \ldots, a_{\ell}\right)$.

For any weight $\lambda$ in $\Lambda$, there exists a unique dominant weight (which is a nonnegative linear combination of $\lambda_{1}, \ldots, \lambda_{\ell}$ ), denoted by $[\lambda]$, such that $\lambda=w[\lambda]$ for some $w$ in $W$.

Lemma 5.1 Given a composition I of $\ell+1$, let $z_{I}=e^{\lambda_{I}}$ where

$$
\lambda_{I}=\sum_{i \in D(I)} \lambda_{i}
$$

Suppose that $w$ lies in $W$ with $D(w) \subseteq D(I)$. Then

$$
\bar{\pi}_{w} z_{I}=e^{w \lambda_{I}}+\sum_{[\lambda]<\lambda_{I}} c_{\lambda} e^{\lambda}, \quad c_{\lambda} \in \mathbb{Z},
$$

where $[\lambda]<\lambda_{I}$ means $\lambda_{I}-[\lambda]$ is a nonnegative linear combination of simple roots and $[\lambda] \neq \lambda_{I}$; moreover, $e^{w \lambda_{I}}$ is a descent monomial if and only if $D(w)=D(I)$. 
Theorem 5.2 The coinvariant algebra $\mathbb{Z}[\Lambda] /\left(a_{1}, \ldots, a_{\ell}\right)$ has a $\mathbb{Z}$-basis

$$
\left\{\bar{\pi}_{w} \bar{z}_{I}: w \in W, D(I)=D(w)\right\}
$$

and decomposes into a direct sum of $\mathscr{H}$-modules

$$
N_{I}=\mathscr{H} \cdot \bar{\pi}_{\alpha(I)} \bar{z}_{I}
$$

as $I$ runs through all compositions of $\ell+1$; moreover, $N_{I}$ is isomorphic to $M_{I} \subseteq \mathscr{H}$ and has a basis $\left\{\bar{\pi}_{w} \bar{z}_{I}: w \in[\alpha(I), \omega(I)]\right\}$. Thus $\mathbb{Z}[\Lambda] /\left(a_{1}, \ldots, a_{\ell}\right)$ is isomorphic to $\mathscr{H}$ as an $\mathscr{H}$-module.

Remark 5.3 Garsia and Stanton [9] pointed out a way to reduce the descent monomials in $\mathbb{Z}[\Lambda]$ to the descent monomials in $\mathbb{Z}[\mathbf{x}]$ for type $A$. However, it does not give Theorem 4.2 directly from Theorem 5.2 , instead, one should consider the Demazure operators on $\mathbb{Z}[X(T)]$ where $X(T)$ is the character group of the maximal torus $T$ of $G L(n, \mathbb{C})$.

\section{Complete flag variety $1_{B}^{G}$ and coinvariants of $(G, B)$}

Let $G$ be a finite group with split $B N$-pair of characteristic $p>0$, whose Weyl group $W$ is generated by $\ell$ simple reflections. Let $1_{B}^{G}=\mathbb{Z}[B \backslash G]$ be the induction of the right trivial representation of $B$ to $G$, i.e. the permutation representation on the right cosets $B \backslash G$.

Given a subset $S \subseteq G$, let $\bar{S}=\sum_{s \in S} s$ in $\mathbb{Z}[G]$. Then $1_{B}^{G} \cong \bar{B} \cdot \mathbb{Z}[G]$ and $\operatorname{End}_{\mathbb{Z}[G]}\left(1_{B}^{G}\right)$ has a basis $\left\{f_{w}: w \in \mathfrak{S}_{n}\right\}$, with $f_{w}$ given by

$$
f_{w}(\bar{B})=\overline{B w B}=\bar{U}_{w} w \bar{B}
$$

where $U_{w}$ is the product of root subgroups $U_{\alpha}$ with $\alpha>0, w^{-1}(\alpha)<0$ [3. Proposition 1.7]. The endomorphism ring $\operatorname{End}_{\mathbb{Z}[G]}\left(1_{B}^{G}\right)$ is isomorphic to the Hecke algebra of $W$ with parameters $q_{i}=\left|U_{s_{i}}\right|$, since the relations satisfied by $\left\{f_{w}\right\}$ are the same as those satisfied by the standard basis $\left\{T_{w}\right\}$. It follows that the 0 -Hecke algebra $\mathscr{H}$ of type $W$ acts on $1_{B}^{G} \otimes \mathbb{F}_{p}$ by 6 . Dually, the left cosets of $B$ give rise to a right $\mathscr{H}$-action. See Kuhn [11] for details.

Since we are mainly concerned with the 0 -Hecke algebra, we shall write $1_{B}^{G}=1_{B}^{G} \otimes \mathbb{F}_{p}$ for simplicity, and similar for $1_{P_{I}}^{G}$ where $P_{I}=B W_{D(I)^{c}} B$ is the parabolic subgroup of $G$ indexed by the composition $I$ of $\ell+1$. For type $A$, one has $G=G L\left(n, \mathbb{F}_{q}\right)$ and $P_{I}$ is the group of all upper triangular block matrices with invertible diagonal blocks of sizes given by the parts of $I$.

To determine the simple factors of an $\mathscr{H}$-module, we develop the following lemma, where the (graded) characteristic is a natural extension of that of type $A$, with the $F_{I}$ 's simply being independent variables.

Lemma 6.1 Given a finite dimensional graded $\mathscr{H}$-module $Q$, let $Q_{I}$ be the submodule of elements that are annihilated by all $T_{j}$ with $j \notin D(I)$, for any composition $I$ of $\ell+1$. Then

$$
\mathcal{F}_{t}(Q)=\sum_{I} c_{I}(Q) F_{I}
$$

summed over all compositions $I$ of $\ell+1$, where

$$
c_{I}(Q)=\sum_{J: D(J) \subseteq D(I)}(-1)^{\ell(I, J)} \operatorname{Hilb}\left(Q_{J}, t\right) .
$$


Define the $q$-ribbon number of type $G$ to be

$$
r_{I}(G)=\sum_{w \in W: D(w)=D(I)}\left|U_{w}\right| .
$$

The characteristic of $1_{B}^{G}$ can be obtained by using Lemma 6.1 and the observations $\left(1_{B}^{G}\right)_{I}=1_{P_{I}}^{G}$,

$$
\left|G / P_{I}\right|=\sum_{w \in W: D(w) \subseteq D(I)}\left|U_{w}\right| .
$$

Theorem 6.2 $\mathcal{F}\left(1_{B}^{G}\right)=\sum_{I} r_{I}(G) F_{I}$.

If $G$ is a finite group of Lie type over a finite field $\mathbb{F}_{q}$, then $\left|U_{w}\right|=q^{\ell(w)}$. In particular, for type $A$, i.e. when $G=G L\left(n, \mathbb{F}_{q}\right)$, one has $r_{I}(G)=r_{I}(q)$. Furthermore, $G$ acts on $\mathbb{F}_{q}[\mathbf{x}]$ and the $H_{n}(0)$-action on $1_{B}^{G}$ induces an $H_{n}(0)$-module structure on

$$
\operatorname{Hom}_{\mathbb{F}_{q}[G]}\left(1_{B}^{G}, \mathbb{F}_{q}[\mathbf{x}]\right) \cong \mathbb{F}_{q}[\mathbf{x}]^{B}
$$

which is $\mathbb{F}_{q}[\mathbf{x}]^{G}$-linear, hence inducing an $H_{n}(0)$-module structure on $\mathbb{F}_{q}[\mathbf{x}]^{B} /\left(\mathbb{F}_{q}[\mathbf{x}]_{+}^{G}\right)$. Explicitly,

$$
T_{w}(f)=\bar{U}_{w} w f, \text { for all } f \text { in } \mathbb{F}_{q}[\mathbf{x}]^{B} .
$$

We observe that

$$
\left(\mathbb{F}_{q}[\mathbf{x}]^{B} /\left(\mathbb{F}_{q}[\mathbf{x}]_{+}^{G}\right)\right)_{I}=\left\{\bar{f}: f \in \mathbb{F}_{q}[\mathbf{x}]^{P_{I}}\right\} .
$$

It follows from Lemma 6.1 that

Theorem 6.3 If $G=G L\left(n, \mathbb{F}_{q}\right)$ and $B$ is the Borel subgroup of $G$, then

$$
\mathcal{F}_{t}\left(\mathbb{F}_{q}[\mathbf{x}]^{B} /\left(\mathbb{F}_{q}[\mathbf{x}]_{+}^{G}\right)\right)=\sum_{I} r_{I}(q, t) F_{I}
$$

We conclude this section with a description of the homology representation $\chi_{q}^{I}$ using the $(G, \mathscr{H})$ bimodule structure on $1_{B}^{G}$, which is now spanned by left $B$-cosets. Smith [16] showed the following left $G$-module decomposition

$$
1_{B}^{G}=\bigoplus_{J} \chi_{q}^{J} .
$$

On the other hand, the decomposition of the 0-Hecke algebra

$$
\mathscr{H}=\bigoplus_{I} \mathscr{H} \cdot T_{\alpha(I)} T_{\alpha(\bar{I} \sim)}^{\prime}
$$

implies a unique way to write

$$
1=\sum_{I} h_{I} T_{\alpha(I)} T_{\alpha(\bar{I} \sim)}^{\prime}, h_{I} \in \mathscr{H} .
$$

By the right action of $\mathscr{H}$ on $1_{B}^{G}$, one has

$$
1_{B}^{G}=\sum_{I} 1_{B}^{G} h_{I} T_{\alpha(I)} T_{\alpha(\bar{I} \sim)}^{\prime}
$$

as a left $G$-module. We show that this is the same as the decomposition (7), that is, 


\section{Theorem 6.4}

$$
1_{B}^{G} h_{I} T_{\alpha(I)} T_{\alpha(\bar{I} \sim)}^{\prime}=\chi_{q}^{I} .
$$

One sees from the above equality that the left $G$-module $\chi_{q}^{I}$ is in general not a right $\mathscr{H}$-module; however, the trivial $G$-representation $\chi_{q}^{(n)}$ and the Steinberg representation $\chi_{q}^{\left(1^{n}\right)}$ are right (isotypic) $\mathscr{H}$-modules.

\section{Remaining Questions}

The equidistribution of inv and maj was first proved on permutations of multisets by P.A. MacMahon in the 1910s; applying an inclusion-exclusion would give their equidistribution on inverse descent classes of $\mathfrak{S}_{n}$. However, the first proof for the latter result appearing in the literature was by Foata and Schützenberger [7] in 1970, using a bijection constructed earlier by Foata [6]. Is there an algebraic proof from the $(q, t)-$ bigraded characteristic 3 of $\mathbb{Z}[\mathbf{x}] /\left(\mathbb{Z}[\mathbf{x}]_{+}^{\mathfrak{S}_{n}}\right)$, which involves inv, maj, and inverse descents?

The next question is on the $\mathscr{H}$-module $1_{B}^{G}$; its simple factors are given by Theorem 6.2 but its decomposition into indecomposable submodules is not obtained in general. Assume $G=G L\left(n, \mathbb{F}_{q}\right)$ below. Recall that

$$
H_{3}(0)=M_{3} \oplus M_{21} \oplus M_{12} \oplus M_{111}
$$

where $M_{I}$ is the indecomposable projective $H_{3}(0)$-module indexed by the composition $I$. For $n=3$ we have candidates for a $q$-analogous $H_{3}(0)$-module decomposition

$$
1_{B}^{G} \cong M_{3} \oplus\left(M_{21} \oplus M_{12}\right)^{\oplus\left(\begin{array}{c}
q+1 \\
2
\end{array}\right)} \oplus\left(M_{111}\right)^{\oplus q^{3}}
$$

which has been checked correct for $q=2,3,5,7$, showing that $1_{B}^{G}$ is a projective $H_{3}(0)$-modules in these cases. Is this true for all primes $q$ ?

On the other hand, for $n=3, q=4,8$, and $n=4, q=2,3$, computations show that $1_{B}^{G}$ is not projective, although the characteristic of $1_{B}^{G}$ is always symmetric. In fact, using the RSK correspondence one can show that

$$
\mathcal{F}\left(1_{B}^{G}\right)=\sum_{\lambda \vdash n} q^{b(\lambda)} \frac{[n] !_{q}}{\prod_{u \in \lambda}\left[h_{u}\right]_{q}} s_{\lambda} .
$$

where $h_{u}$ is the hook length of the box $u$ in the Young diagram of $\lambda$ and $b(\lambda)=\lambda_{2}+2 \lambda_{3}+3 \lambda_{4}+\cdots$.

Computations also show that $\mathbb{F}_{q}[\mathbf{x}]^{B} /\left(\mathbb{F}_{q}[\mathbf{x}]_{+}^{G}\right)$ is not projective for $n=3, q=2,3$. Besides being curious to know its decomposition, we wonder if there is any $q$-analogue of the Demazure operators, which might give another $H_{n}(0)$-action on $\mathbb{F}_{q}[\mathbf{x}]^{B} /\left(\mathbb{F}_{q}[\mathbf{x}]_{+}^{G}\right)$.

\section{Acknowledgements}

The author is grateful to Victor Reiner for asking questions which led to the research and for giving many constructive suggestions. He is indebted to Andrew Berget, Tom Denton, Anne Schilling, and Monica Vazirani for interesting conversations and hospitality during the visit to UC Davis. He thanks Marcelo Aguiar, Sami Assaf, Nantel Bergeron, Adriano Garsia, Patricia Hersh, Liping Li, Sarah Mason, Dennis Stanton, Peter Webb, and two anonymous referees for their helpful suggestions and comments. 


\section{References}

[1] E.E. Allen, The descent monomials and a basis for the diagonally symmetric polynomials, J. Algebraic Combin. 3 (1994) 5-16.

[2] M. Demazure, Désingularisation des variétés de Schubert généralisés, Ann. Sci. École Norm. Sup. 7 (1974) 53-88.

[3] F. Digne and J. Michel, Representations of Finite Groups of Lie Type, London Math. Soc. Student Texts 21, Cambridge University Press (1991).

[4] G. Duchamp, D. Krob, B. Leclerc, and J.-Y. Thibon, Fonctions quasi-symétriques, fonctions symétriques non commutatives et algèbres de Hecke à $q=0$. C.R. Acad. Sci. Paris 322 (1996) $107-112$.

[5] M. Fayers, 0-Hecke algebras of finite Coxeter groups, J. Pure Appl. Algebra 199 (2005) $27-41$.

[6] D. Foata, On the Netto inversion number of a sequence, Proc. Amer. Math. Soc. 19 (1968) 236-240.

[7] D. Foata and M.-P. Schützenberger, Major index and inversion number of permutations, Math. Nachr. 83 (1970), 143-159.

[8] A. Garsia, Combinatorial methods in the theory of Cohen-Macaulay rings, Adv. Math. 38 (1980) 229-266.

[9] A. Garsia and D. Stanton, Group actions of Stanley-Reisner rings and invariant of permutation groups, Adv. Math. 51 (1984) 107-201.

[10] D. Krob and J.-Y. Thibon, Noncommutative symmetric functions IV: Quantum linear groups and Hecke algebras at $q=0$, J. Algebraic Combin. 6 (1997) 339-376.

[11] N. Kuhn, The modular Hecke algebra and Steinberg representation of finite Chevalley groups, J. Algebra 91 (1984), 125-141.

[12] S. Kumar, Demazure character formula in arbitrary Kac-Moody setting, Invent. Math. 89 (1987) 395-423.

[13] S. Mason, An explicit construction of type A Demazure atoms. J. Algebraic Combin. 29 (2009), 295-313.

[14] P.N. Norton, 0-Hecke algebras, J. Austral. Math. Soc. A 27 (1979) 337-357.

[15] V. Reiner and D. Stanton, $(q, t)$-analogues and $G L_{n}\left(\mathbb{F}_{q}\right)$, J. Algebr. Comb. 31 (2010) $411-454$.

[16] S.D. Smith, On decomposition of modular representations from Cohen-Macaulay geometries, J. Algebra 131 (1990) 598-625.

[17] R. Steinberg, On a theorem of Pittie, Topology 14 (1975) 173-177. 